\title{
A RESULT ON TWO-DIMENSIONAL POLAR LATTICES
}

\author{
T. P. DE SILVA
}

Department of Matbematics; University of Sri Jayawardenepura, Nugegoda, Sri Lanka.

(Date of receipt : 28.05.85)

(Date of acceptance : 18.12.86)

\begin{abstract}
Suppose $\mathrm{P}$ and $\mathrm{P}^{\mathrm{O}}$ denote closed positive and open positive quadrants in $R^{2}$ respectively. Let $\Lambda$ he any lattice in $R^{2}$ with polar lattice $\Lambda^{*}$. Let $F$ be a convex and symmetric (with respect to the axes of coordinates) distance function with $\mathrm{F}(1,0)=\mathrm{F}(0, \mathrm{t})=1$, where $\mathrm{t} \in \mathrm{R}$ and let $\mu=$ area $\left(\mathrm{x} \in \mathrm{R}^{2} / \mathrm{F}(\mathrm{x}) \leqslant 1\right)$. For certain distance functions $F$, there exist non-zero $\underline{x} \in \mathrm{P} \cap \bar{\Lambda}$ and $\underline{y} \in \mathrm{P} O \mathrm{P} \cap \Lambda^{*}$ such that $\mu \mathrm{F}(\mathrm{x}) \mathrm{F}(\mathrm{y}) \leqslant \gamma_{\mathrm{t}}$, where $\gamma_{\mathrm{t}}$ is a constant depending on $\mathrm{t}$ and the distance function. There exist a lower bound $2(t+1 / t)$ and an upper bound $4(t+1 / t)$ for $\gamma_{t}$ over all convex symmetric distance functions.
\end{abstract}

\section{Introduction}

Let $\mathrm{P}$ and $\mathrm{P}^{\mathrm{O}}$ denote closed positive and open positive quadrants in $\mathrm{R}^{2}$ respectively. Let $\Lambda$ be any lattice in $R^{2}$ with polar lattice $\Lambda^{*}$. Let $\mathrm{F}$ be a convex and symmetric (with respect to the axes of coordinates) distance function with $F(1,0)=F(0, t)=1$, where $t \in R$. Without loss of generality we can take $t \geqslant 1$. If $t \leqslant 1$, we have the same situation as in the case when $t \geqslant 1$ with the coordinate axes interchanged. Let $\mu=\operatorname{area}\left\{\underline{x} \in R^{2} / f(\underline{x}) \leqslant 1\right\}$.

Hossain and Worley ${ }^{3}$ have shown that for certain distance functions $F$, there exist non-zero $\underline{x} \in \mathrm{P} \cap \Lambda$ and $\underline{\mathrm{y}} \in \mathrm{P}^{\mathrm{O}} \cap \Lambda^{*}$. such that

$$
\mu \mathrm{F}(\underline{\mathrm{x}}) \mathrm{F}(\underline{\mathrm{y}}) \leqslant \gamma_{\mathrm{t}},
$$

where $\gamma_{t}$ is a constant depending on $t$ and the distance function. In this note we show that $\gamma_{t}$ has a lower bound and an upper bound over all the convex symmetric distance functions. In this note symmetric means the symmetricity with respect to the axes of coordinates.

\section{Discussion}

The following notations will be used frequently in this section.

$$
\begin{aligned}
& \mathrm{F}_{1}(\Lambda)=\inf \{\mathrm{F}(\underline{\mathrm{x}}): \underline{\mathrm{x}} \in \Lambda \cap \mathrm{P}\} \\
& \mathrm{F}_{\mathbf{2}}\left(\Lambda^{*}\right)=\inf \left\{\mathrm{F}\left(\underline{x}^{*}\right): \underline{x}^{*} \in \Lambda^{*} \cap \mathrm{D}^{\mathrm{O}}\right\},
\end{aligned}
$$

where $\mathrm{F}$ is a distance function. 


\section{Theorem:} then

If $F$ is any convex symmetric distance function with $F(1,0)=F(0, t)=1$,

$$
2(t+1 / t) \leqslant \mu F_{1}(\Lambda) F_{2}\left(\Lambda^{*}\right) \leqslant 4(t+1 / t)
$$

for the lattice $\Lambda$ with basis $\{(1,0),(0, t)\}$

The lower bound is best possible for the distance function $F\left(x_{1}, x_{2}\right)=$ $\left|\mathrm{x}_{1}\right|+1 / \mathrm{t}\left|\mathrm{x}_{2}\right|$ and the lattice $\Lambda$ with a basis $(1,0)$ and $(0, t)$. The upper bound may not be best possible, but cannot be below $4 \mathrm{t}$. In order to prove the theorem, we use the following lemmas. ${ }^{2}$

\section{Lemma 1}

Let $\Lambda$ be the lattice with a basis $\{(1,0),(0, t)\}$. Then

$\min _{\alpha} \mu \mathrm{F}_{1}(\Lambda) \mathrm{F}_{2}\left(\Lambda^{*}\right)=2(\mathrm{t}+1 / \mathrm{t})$, for the convex symmetric polygonal distance function $\mathrm{F}$ given by

$\mathrm{F}\left(\mathrm{x}_{1}, \mathrm{x}_{2}\right)=\max \left\{\frac{1-\alpha}{\alpha}\left|\mathrm{x}_{1}\right|+1 / \mathrm{t}\left|\mathrm{x}_{2}\right|,\left|\mathrm{x}_{1}\right|+\frac{1-\alpha}{\alpha \mathrm{t}}\left|\mathrm{x}_{2}\right|\right\}$

where $1 / 2 \leqslant \alpha \leqslant 1$.

( $\alpha$ has to satisfy the above conditions since $\mathrm{F}$ is convex and symmetric).

\section{Lemma 2}

Let $\Lambda$ be a lattice with basis $\{(1,0),(0, t)\}$. Let $F$ be the convex polygonal distance function, where $\mathrm{F}\left(\mathrm{x}_{1}, \mathrm{x}_{2}\right)=1$ has two more vertices at $(\alpha, \alpha \mathrm{t})$ and $(\beta, \beta / \mathrm{t})$ in $\mathrm{P}^{\mathrm{O}}$ in addition to $(1,0)$ and $(0, t)$, where $1 / 2 \leqslant \alpha \leqslant 1$ and the limit of $\beta$ depends on $\alpha$.

$$
\text { Then } \min _{\alpha, \beta} \mu \mathrm{F}_{1}(\Lambda) \mathrm{F}_{2}\left(\Lambda^{*}\right) \geqslant 2(\mathrm{t}+1 / \mathrm{t})
$$

From Lemma 1 and Lemma 2, we can establish the left hand side of the inequality in the theorem.

Suppose $\mathrm{F}\left(\mathrm{x}_{1}, \mathrm{x}_{2}\right)=1$ intersects the

lines $\mathrm{OL}$ at $\mathrm{B}$ and $\mathrm{OM}$ at $\mathrm{C}$ respectively,

where $\mathrm{L} \equiv(1, \mathrm{t})$ and $\mathrm{M}=(1,1 / \mathrm{t})$.

Let $\mathrm{B} \equiv(\alpha, \alpha \mathrm{t})$ and $\mathrm{C} \equiv(\beta, \beta / \mathrm{t})$.

The curve $\mathrm{F}\left(\mathrm{x}_{1}, \mathrm{x}_{2}\right)=1$ passes through

the points $A \equiv(0, t)$ and $D \equiv(1,0)$.

Then $\mathrm{F}_{1}(\Lambda)=1$. 


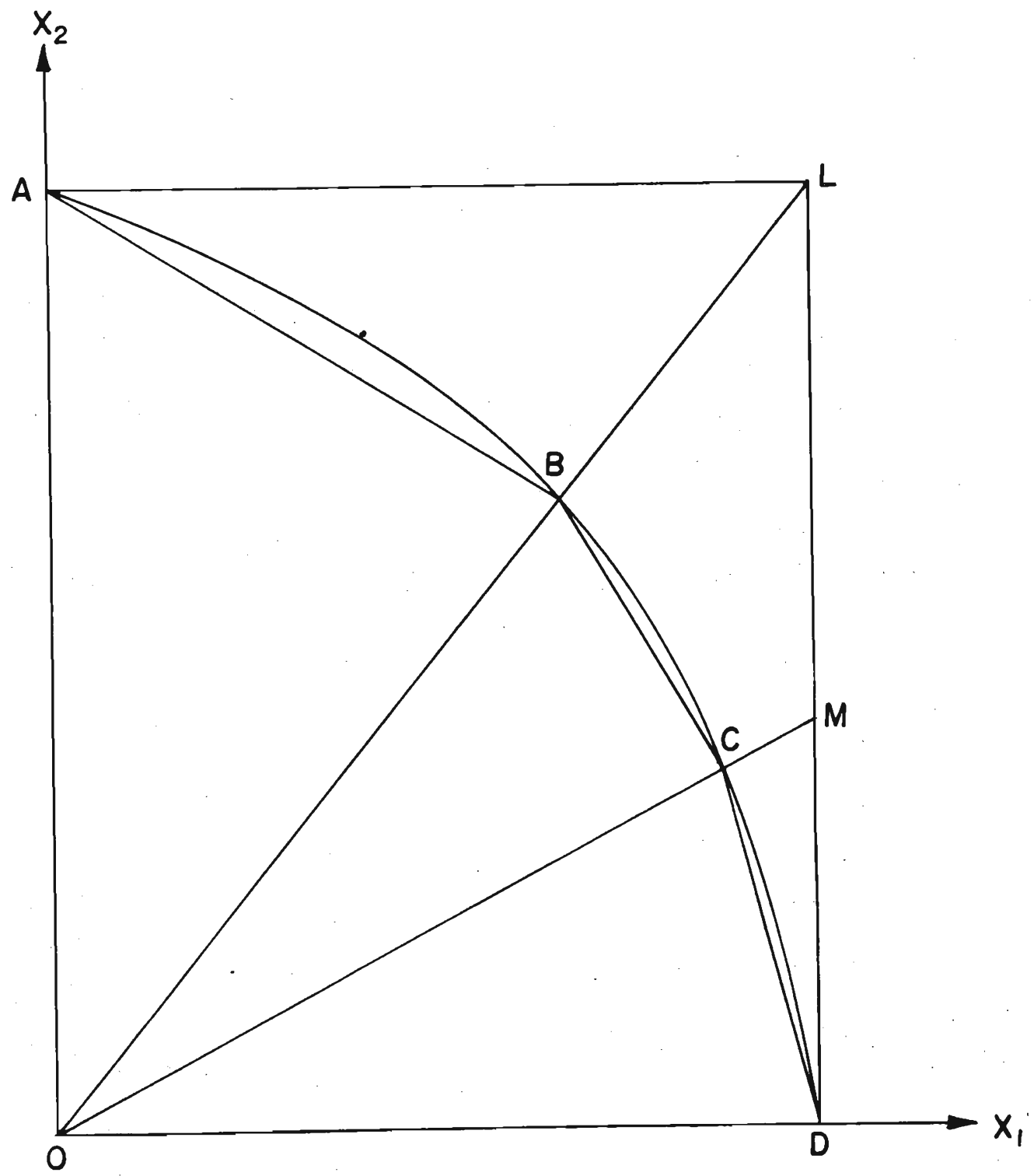

Figure 1. The curve $F\left(x_{1}, x_{2}\right)=1$ passes through the points $A=(0, t)$ and $D=(1,0)$. It intersects the lines $O L$ at $B$ and $O M$ at $C$ respectively, where $L=(1, t)$ and $M=(1,1 / t)$. 
and $\mathrm{F}_{2}\left(\Lambda^{*}\right)=\mathrm{F}(1,1 / \mathrm{t})=1 / \beta \mathrm{F}(\beta, \beta / \mathrm{t})=1 / \beta$

Let $\mathrm{G}\left(\mathrm{x}_{1}, \mathrm{x}_{2}\right)=1$ be the equation of the polygonal arc ABCD. Then for this distance function $\mathrm{G}$,

$$
\mathrm{G}_{1}(\Lambda)=1 \text { and } \mathrm{G}_{2}\left(\Lambda^{*}\right)=1 / \beta
$$

let $\mu_{\mathrm{G}}=4 \mathrm{x}$ area $\{$ polygon OABCD.

Then from. Lemma 2 we have

$\mu_{\mathrm{g}} \mathrm{G}_{1}(\Lambda) \mathrm{G}_{2}\left(\Lambda^{*}\right)=\mu_{\mathrm{G}} \cdot 1 / \beta \geqslant 2(\mathrm{t}+1 / \mathrm{t})$ for all suitable $\alpha$ and $\beta$.

Now let $\mu=\operatorname{area}\left\{\underline{x} \in R^{2}: F(\underline{x}) \leqslant 1\right\}$.

Then from the convexity $\mu \geqslant \mu_{\mathrm{G}}$.

Hence $\mu F_{1}(\Lambda) F_{2}\left(\Lambda^{*}\right)=\mu .1 / \beta \geqslant \mu_{G} \cdot 1 / \beta \geqslant 2(t+1 / t)$.

ie we have proved that

$\mu \mathrm{F}_{1}(\Lambda) \quad \mathrm{F}_{2}\left(\Lambda^{*}\right) \geqslant 2(\mathrm{t}+1 / \mathrm{t})$ for all convex symmetric distance functions $F$ and the lattice $\Lambda$ with basis $(1,0)$ and $(0, t)$.

$\therefore \min _{\mathrm{F}} \mu \mathrm{F}_{1}(\Lambda) \mathrm{F}_{2}\left(\Lambda^{*}\right) \geqslant 2(\mathrm{t}+1 / \mathrm{t})$.

Now we proceed to prove the right hand side of the inequality in the theorem.

Let $\Lambda$ be any two dimensional lattice and $(a, b) \in \Lambda \cap \mathrm{P}^{\mathrm{O}}$ and $(-c, d) \in \Lambda \cap Q$ be two points such that $F(a, b)$ and $F(d, c)$ are minimal, where $\mathbf{Q}$ is the open second quadrant.

Note that if $(-c, d) \in \Lambda$, then $\frac{(d, c)}{d(\Lambda)} \in \Lambda^{*}$, where $d(\Lambda)$ is the determinant of $\Lambda$.

Let $\mathrm{D}=\left\{\left(\mathrm{x}_{1}, \mathrm{x}_{2}\right) \in \mathrm{R}^{2}: \mathrm{F}\left(\mathrm{x}_{1}, \mathrm{x}_{2}\right) \leqslant \mathrm{F}(\mathrm{a}, \mathrm{b}), \mathrm{F}\left(-\mathrm{x}_{2}, \mathrm{x}_{1}\right) \leqslant \mathrm{F}(\mathrm{d}, \mathrm{c})\right\}$.

Then from the choice of $(a, b)$ and $(-c, d)$ there are non-zero points of $\Lambda$ in $D$.

Then there are no non-zero points of $\Lambda$ in the parallelogram.

$\left\{\left(\mathrm{x}_{1}, \mathrm{x}_{2}\right) \in \mathrm{R}^{2}:\left|-\bar{x}_{1}+\mathrm{tx} \mathrm{x}_{2}\right| \leqslant \mathrm{t} \mathrm{F}(\mathrm{d}, \mathrm{c}),\left|\mathrm{tx} \mathrm{x}_{1}+\mathrm{x}_{2}\right| \leqslant \mathrm{t} \mathrm{F}(\mathrm{a}, \mathrm{b})\right\}$

which lies entirely in $\mathrm{D}$.

Hence by Minkowski's linear form theorem ${ }^{1}$, we have

$t^{2} F(a, b) F(d, c) \leqslant\left(1+t^{2}\right) d(\Lambda)$ 
$\therefore F(a, b) \frac{F(d, c)}{d(\Lambda)} \leqslant 1+1 / t^{2}$

Now $\mu=\operatorname{area}\left\{\left(\mathrm{x}_{1}, \mathrm{x}_{2}\right) \in \mathrm{R}^{2}: \mathrm{F}\left(\mathrm{x}_{1}, \mathrm{x}_{2}\right) \leqslant 1\right\}$

$\leqslant 4 \mathrm{t}$, as $\mathrm{F}\left(\mathrm{x}_{1}, \mathrm{x}_{2}\right)$ is convex and symmetric.

$\therefore \mu \mathrm{F}_{1}(\Lambda) \mathrm{F}_{2}\left(\Lambda^{*}\right) \leqslant 4(\mathrm{t}+1 / \mathrm{t})$.

\section{References:}

1. CASSELS, J. W. S., (1971) "Introduction to Geometry of Numbers" Second edition, Springer - Verlag.

2. DE SILVA, T. P., (1977) "Results on Polar Lattices" Thesis submitted for the degree of Master of Science of Monash University.

3. HOSSAIN, F. \& WORLEY, R. T., 'Positive Points in Polar Lattices" (Personal Communication). 